\title{
Aplicación de un Método Iterativo de dos Pasos para el Cálculo de Flujos de Potencia
}

\author{
Rubén Villafuerte ${ }^{(1) \star}$, Rubén. A. Villafuerte ${ }^{(2)}$, Jesús Medina ${ }^{(1)}$ y Edgar Mejía ${ }^{(1)}$ \\ (1) Universidad Veracruzana, Facultad de Ingeniería Campus ciudad Mendoza, Ver., México \\ (e-mail:rubenv46@yahoo.com.mx; jemedina@uv.mx; edgar_posgrado@hotmail.com) \\ (2) Instituto Tecnológico Nacional Campus Orizaba Ver., México (e-mail: dreyko999@gmail.com)
}

* Autor a quien dirigir la correspondencia

Recibido Jul. 21, 2016; Aceptado Sep. 22, 2016; Versión final Nov. 3, 2016, Publicado Feb. 2017

\begin{abstract}
Resumen
Este trabajo presenta un método iterativo para resolver las ecuaciones de flujo de potencia a partir del establecimiento de funciones no lineales en cada nodo. Con el método propuesto se generan $\mathrm{N}-1$ funciones no lineales, las cuales se resuelven iterativamente hasta lograr la convergencia en los voltajes de cada nodo del sistema eléctrico de potencia. El análisis de convergencia demostró que el método iterativo de dos pasos es de cuarto orden. En este trabajo se utiliza la fórmula original del método de NewtonRaphson en el primer paso y un método tipo Halley en el segundo paso. Los sistemas de prueba de 14, 30 y 57 nodos son simulados para conocer las características del método propuesto. Entre las fortalezas del método se puede mencionar que no requiere de una programación sofisticada y que representa una opción más para calcular flujos de potencia.
\end{abstract}

Palabras clave: método iterativo; método de Newton-Raphson; método de Halley; sistemas eléctricos de potencia

\section{Application of a Two Steps Iterative Method for Power Flow Calculation}

\begin{abstract}
This paper presents an iterative method for solving the power flow equations, starting from the establishment of non-linear functions on each node. With the proposed method, $\mathrm{N}-1$ nonlinear functions are generated, which are iteratively solved to achieve convergence at voltages of each node in the power system. The convergence analysis showed that the two-step iterative method is fourth order. The original formula of Newton-Raphson method in the first step and Halley-type method in the second step is used in this work. Test systems with 14, 30 and 57 nodes are simulated to determine the characteristics of the proposed method. Among the strengths of the method it may be mentioned that it does not require sophisticated programming and that it represents an additional method for calculating power flows.
\end{abstract}

Keywords: electric power systems; iterative method; Halley method; Newton-Raphson method 


\section{INTRODUCCIÓN}

En el análisis de redes eléctricas el cálculo de la potencia real y reactiva es fundamental para conocer la condición de operación bajo una carga conocida. Para el cálculo de flujos de potencia se establecen ecuaciones no lineales que se resuelven preferentemente de una forma iterativa. Para ello investigadores (Tinney et al., 1967; Stott et., al 1974; y Nanda et al., 1992) trabajaron con modelos provenientes del método de Newton-Raphson. Así mismo, se han desarrollado métodos con los cuales se intenta aproximar las ecuaciones de flujos de potencia en series Taylor con la finalidad de obtener la solución exacta, Sauer et al. (1981). El método de Newton-Raphson consiste en resolver un sistema de ecuaciones que inicialmente son no lineales. La linealización se lleva a cabo por medio del cálculo de las derivadas parciales de la potencia real y reactiva con respecto de la magnitud del voltaje y su ángulo, o en función de las componentes cartesianas de los voltajes en cada nodo de la red, dependiendo de la versión de NewtonRaphson utilizada. La aplicación del método de Newton-Raphson a la solución de las ecuaciones de flujos de potencia genera la matriz jacobiana que tiene, para un sistema con únicamente nodos de carga un orden de $2^{*}(\mathrm{~N}-1)$ (Tinney et al., 1967; Stagg et al., 1968). El sistema a resolver en este caso, tiene la estructura de la ecuación (1).

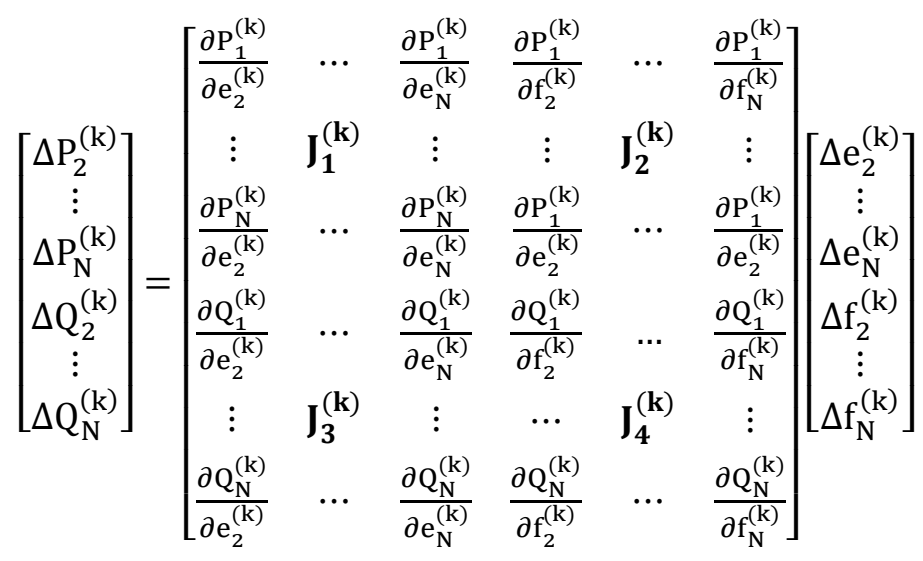

En donde: $\Delta P_{i}^{(k)}$ Es el incremento de la potencia real ante un cambio en el voltaje del nodo $i$ del sistema de potencia en la iteración $k ; \Delta Q_{i}^{(k)}$ Es el incremento de la potencia reactiva ante un cambio en el voltaje del nodo $i$ del sistema de potencia en la iteración $k ; \Delta e_{i}^{(k)}$ Es el incremento de la parte real del voltaje en cada nodo del sistema eléctrico de potencia; $\Delta f_{i}^{(k)}$ Es el incremento de la parte imaginaria del voltaje en cada nodo del sistema eléctrico de potencia; ei Es la componente real del voltaje del nodo i; fi Es la componente imaginaria del voltaje del nodo $\mathrm{i} ; J_{1}^{(k)}$ Es la matriz de derivadas de la potencia real con respecto a la parte real del voltaje de los nodos del sistema de potencia en la iteración $k ; J_{2}^{(k)}$ Es la matriz de derivadas de la potencia real con respecto a la parte imaginaria del voltaje de los nodos del sistema de potencia en la iteración $k ; J_{3}^{(k)}$ Es la matriz de derivadas de la potencia reactiva con respecto a la parte real del voltaje de los nodos del sistema de potencia en la iteración $k$, y $J_{4}^{(k)}$ Es la matriz de derivadas de la potencia reactiva con respecto a la parte imaginaria del voltaje de los nodos del sistema de potencia en la iteración $k$.

Las submatrices $\mathrm{J}_{1}, \mathrm{~J}_{2}, \mathrm{~J}_{3}$ y $\mathrm{J}_{4}$, representan a la matriz jacobiana del método de Newton-Raphson y los voltajes de cada nodo del sistema eléctrico de potencia se corrigen calculando las componentes rectangulares en forma iterativa con las ecuaciones (2) (Stagg et al., 1968; Grainger et al., 1999; Zhu, 2009).

$$
\begin{gathered}
e_{i}^{(k+1)}=e_{i}^{(k)}+\Delta e_{i}^{(k)} \\
\mathrm{f}_{\mathrm{i}}^{(\mathrm{k}+1)}=\mathrm{f}_{\mathrm{i}}^{(\mathrm{k})}+\Delta \mathrm{f}_{\mathrm{i}}^{(\mathrm{k})}
\end{gathered}
$$

El sistema representado por la ecuación (1) y otros derivados del método de Newton-Raphson han sido analizado por investigadores entre los cuales se encuentran a (Hale H. et al., 1959; y Tinney et al., 1967), para resolverlo aplican esquemas de almacenamiento de matrices, métodos de ordenamiento y factorización con el objetivo de reducir el espacio utilizado en computadora digital y el tiempo de ejecución (Tewarson, 1973). El número de iteraciones, independientemente del tamaño del sistema según reportes, está entre 3 y 5 (Tinney et al., 1967; y Stott et., al 1974). En la ecuación (1), $\Delta P_{i}^{(k)}$ y $\Delta Q_{i}^{(k)}$ y las submatrices 
$J_{1}$ a $J_{4}$, son funciones que dependen de los voltajes de cada nodo y de la matriz de admitancias del sistema eléctrico de potencia (Tinney et al., 1967; y Stagg et al., 1968). La versión desacoplada del método de Newton-Raphson (Stagg et al., 1968; Stott et., al 1974), que se deriva de la forma polar permite calcular la magnitud del voltaje y su ángulo en forma separada, que también requiere de la aplicación de técnicas ordenamiento y factorización (Tewarson, 1973). Se han desarrollado otros métodos para calcular flujos de potencia en sistemas de transmisión y en sistemas de distribución, Mamdouh et al. (2015). Así mismo se ha utilizado software comercial como Matlab en donde el método de Newton-Raphson se ha programado para calcular flujos de potencia (Nayak, et al., 2014).

Para el cálculo de las raíces reales de una función no lineal existen diferentes métodos numéricos entre los que destaca la fórmula original de Newton-Raphson representada por la ecuación (3) (Nakamura et al., 1992; y Chapra et al., 2014).

$x_{n+1}=x_{n}-\frac{f\left(x_{n}\right)}{f^{\prime}\left(x_{n}\right)}$

En dónde: $f\left(x_{n}\right)$ Es la función no lineal evaluada en la iteración $n ; f^{\prime}\left(x_{n}\right)$ Es la derivada de la función no lineal evaluada en la iteración $n ; \mathrm{y} \mathrm{x}_{\mathrm{n}}$ es el valor de la raíz en la iteración $n$.

Para la solución de ecuaciones no lineales reales (Homeier et al., 2005; Kou et al., 2007; Petko et al., 2014; y Hueso et al., 2015) proponen adicionar a la ecuación (3), uno o más pasos con el objetivo de reducir el número de iteraciones, incrementar el orden de convergencia y hacer más eficiente al método. Otros métodos que tienen como base a la fórmula original de Newton-Raphson se han propuesto para encontrar las raíces de funciones no lineales, ellos se pueden encontrar en: (Changbum et al., 2009; Mohamed, 2012; Bahgat et al., 2014).

En este trabajo se utiliza un método iterativo tipo Newton para resolver el problema de flujos de potencia, en donde la fórmula original de Newton-Raphson (3) es la base. En el estudio de flujos de potencia, los voltajes de cada nodo son las variables estado que se calculan con la ecuación (1), con la ecuación (3) o con un método de más pasos para elevar el orden de convergencia.

\section{ECUACIONES A RESOLVER}

En un sistema eléctrico de potencia la aplicación de las leyes y teoremas de circuitos eléctricos permiten establecer la relación entre las corriente $l_{i}$ y el voltaje $V_{i}$ en cada nodo de la red, que es representado por la ecuación (4).

$\bar{I}_{l}=\sum_{j=1}^{N} \bar{Y}_{i j} \bar{V}_{j}$

En donde: $\bar{Y}_{i j}$ es el elemento ij de la matriz de admitancias; $\bar{I}_{l}$ es la corriente inyectada el nodo i; y $\bar{V}_{l}$ es el voltaje en el nodo j; y $\mathrm{N}$ es el número de nodos. La barra superior en los elementos de la ecuación (4) indica que son cantidades complejas. La potencia compleja en cada nodo i es representada por la ecuación (5).

$\left(P_{i}+j Q_{i}\right)=\bar{V}_{i} \sum_{j=1}^{N} \bar{Y}_{i j}^{*} \bar{V}_{j}^{*}$

En donde: $P_{i}$ es la potencia real en el nodo $\mathrm{i} ; \mathrm{Q}_{\mathrm{i}}$ es la potencia real en el nodo $\mathrm{i} ; \mathrm{y}$ * significa el conjugado. La ecuación (5) se puede expresar como una función compleja por medio de la ecuación (6).

$f_{i}\left(V_{1}, V_{2},, V_{N}\right)=\left(P_{i}+j Q_{i}\right)-\bar{V}_{i} \sum_{j=1}^{N} \bar{Y}_{i j}^{*} \bar{V}_{j}^{*}$

En el estudio de flujos de potencia es necesario resolver $(\mathrm{N}-1)$ funciones no lineales de la forma representada en la ecuación (6). El método de Newton-Raphson linealiza la ecuación (6) de cada nodo de la red mediante la formación de la matriz jacobiana que tiene una dimensión de $2^{*}(\mathrm{~N}-1)$ y su solución implica la aplicación de esquemas de ordenamiento y factorización. En este trabajo se obtiene la solución del problema de flujos de potencia haciendo uso de la ecuación (6) en combinación con métodos tipo Newton sin utilizar esquemas de programación sofisticados.

\section{DESARROLLO DEL MÉTODO PROPUESTO}

El método que se propone en este trabajo es de dos pasos, el análisis de convergencia demostró que es de cuarto orden. Métodos de cuarto orden para funciones no lineales son desarrollados por Ramandeep, et al. (2014), quienes proponen una familia de fórmulas tipo Chebyshev-Halley para calcular en dos pasos el valor 
de las raíces de funciones no lineales. Métodos de más pasos y de mayor orden se han desarrollado para el cálculo de raíces en funciones reales (Bahgat, et al., 2014), sin embargo aquí se utiliza un método de dos pasos para resolver las funciones complejas de flujos de potencia (6).

El método iterativo de dos pasos de cuarto orden que se propone en este trabajo es representado por las ecuaciones (7) y (8).

$y_{n}=x_{n}-\beta_{o} \frac{f\left(x_{n}\right)}{f^{\prime}\left(x_{n}\right)}$

$x_{n+1}=y_{n}-\frac{\beta_{1} f\left(y_{n}\right)\left(f^{\prime}\left(y_{n}\right)\right)^{2}}{\beta_{2}\left(f^{\prime}\left(y_{n}\right)\right)^{2}+\beta_{3} \alpha_{x}\left(f\left(y_{n}\right)\right)^{3} f^{\prime \prime}\left(y_{n}\right)}$

En la ecuación (7) se modifica la formula de Newton-Raphson con el coeficiente $\beta_{0}$, que es un número real, la ecuación (8) es un método que tipo Halley (Petko et al., 2014), en donde los coeficientes $\beta_{\circ}, \beta_{1}, \beta_{2}, \alpha_{x}$, son números reales. A continuación se hace la prueba de convergencia del método iterativo propuesto, con el programa Derive.

\section{Análisis de convergencia del método iterativo propuesto}

Supóngase que $y$ es una raíz compleja de $f(x)$, por lo que: $f(\gamma)=0$ y $f^{\prime}(Y) \neq 0$ y además $x_{n}=e_{n}+\gamma$, considerando que: $\beta_{\circ}=1, \beta_{1}=\beta_{2}=2, \alpha_{x}=1$, y haciendo uso de la expansión en series de Taylor para la función $f\left(x_{n}\right)$, en la vecindad de $y$ se tiene:

$f\left(x_{n}\right)=f\left(e_{n}+\gamma\right)=f(\gamma)+e_{n} f^{\prime}(\gamma)+\frac{1}{2} e_{n}^{2} f^{\prime \prime}(\gamma)+\frac{1}{3 !} e_{n}^{3} f^{\prime \prime \prime}(\gamma)+\frac{1}{4 !} e_{n}^{4} f^{i v}(\gamma)+\frac{1}{5 !} e_{n}^{5} f^{v}(\gamma)+O\left(e_{n}\right)^{6}$

Debido a que: $f(\gamma)=0$, se tiene:

$f\left(x_{n}\right)=f^{\prime}(\mathrm{Y})\left(e_{n}+\frac{1}{2 f^{\prime}(\mathrm{Y})} e_{n}^{2} f^{\prime \prime}(\mathrm{Y})+\frac{1}{3 ! f^{\prime}(\mathrm{Y})} e_{n}^{3} f^{\prime \prime \prime}(\mathrm{Y})+\frac{1}{4 ! f^{\prime}(\mathrm{Y})} e_{n}^{4} f^{i v}(\mathrm{Y})+\frac{1}{5 ! f^{\prime}(\mathrm{Y})} e_{n}^{5} f^{v}(\mathrm{Y})+O\left(e_{n}\right)^{6}\right)$

Definiendo:

$C_{k}=\frac{f^{k}(\gamma)}{k ! f^{\prime}\left(f^{\prime}(\mathrm{y})\right)}$ Para $\mathrm{k}=2,3,4,5, .$.

Se tiene que:

$f\left(x_{n}\right)=f^{\prime}(\mathrm{Y})\left(e_{n}+C_{2} e_{n}^{2}+C_{3} e_{n}^{3}+C_{4} e_{n}^{4}+C_{5} e_{n}^{5}+C_{6} e_{n}^{6}+O\left(e_{n}\right)^{7}\right)$

Derivando sucesivamente con respecto a $e_{n}$, se tiene:

$f^{\prime}\left(x_{n}\right)=f^{\prime}(\mathrm{Y})\left(1+2 C_{2} e_{n}+3 C_{3} e_{n}^{2}+4 C_{4} e_{n}^{3}+5 C_{5} e_{n}^{4}+6 C_{6} e_{n}^{5}+O\left(e_{n}\right)^{6}\right)$

$f^{\prime \prime}\left(x_{n}\right)=f^{\prime}(\mathrm{Y})\left(2 C_{2}+6 C_{3} e_{n}+12 C_{4} e_{n}^{2}+20 C_{5} e_{n}^{3}+30 C_{6} e_{n}^{4}+O\left(e_{n}\right)^{5}\right)$

$f^{\prime \prime \prime}\left(x_{n}\right)=f^{\prime}(\mathrm{y})\left(6 C_{3}+24 C_{4} e_{n}+60 C_{5} e_{n}^{2}+120 C_{6} e_{n}^{3}+O\left(e_{n}\right)^{4}\right)$

$f^{i v}\left(x_{n}\right)=f^{\prime}(\mathrm{Y})\left(24 C_{4}+120 C_{5} e_{n}+360 C_{6} e_{n}^{2}+O\left(e_{n}\right)^{3}\right)$

Considerando que $\beta o=1$, de la ecuación (7), y desarrollando $\frac{f\left(x_{n}\right)}{f^{\prime}\left(x_{n}\right)}$ en series de Taylor con el programa derive:

$\frac{f\left(x_{n}\right)}{f^{\prime}\left(x_{n}\right)}=e_{n}-C_{2} e_{n}^{2}+\left(2 C_{3}-2 C_{2}^{2}\right) e_{n}^{3}+e_{n}^{4}\left(4 C_{2}^{3}-7 C_{2} C_{3}+3 C_{4}\right)+(O) e_{n}^{5}$

Definiendo: 
$d_{n}=-\frac{f\left(x_{n}\right)}{f^{\prime}\left(x_{n}\right)}=-\left(e_{n}-C_{2} e_{n}^{2}+\left(2 C_{3}-2 C_{2}^{2}\right) e_{n}^{3}+e_{n}^{4}\left(4 C_{2}^{3}-7 C_{2} C_{3}+3 C_{4}\right)+(O) e_{n}^{5}\right)$

Sustituyendo la ecuación (16) en la ecuación (7):

$y_{n}=C_{2} e_{n}^{2}+\left(2 C_{3}-2 C_{2}^{2}\right) e_{n}^{3}+e_{n}^{4}\left(4 C_{2}^{3}-7 C_{2} C_{3}+3 C_{4}\right)+(O) e_{n}^{5}$

Desarrollando en series de Taylor $f\left(y_{n}\right)$ cerca de $d n$ :

$f\left(y_{n}\right)=f\left(x_{n}-\frac{f\left(x_{n}\right)}{f^{\prime}\left(x_{n}\right)}\right)=f\left(x_{n}\right)+f^{\prime}\left(x_{n}\right) d_{n}+\frac{1}{2} f^{\prime \prime}\left(x_{n}\right) d_{n}^{2}+\frac{1}{3 !} f^{\prime \prime \prime}\left(x_{n}\right) d_{n}^{3}+\frac{1}{4 !} f^{(i v)}\left(x_{n}\right) d_{n}^{4}+(O) d_{n}^{5}$

$f\left(y_{n}\right)=f\left(x_{n}-\frac{f\left(x_{n}\right)}{f^{\prime}\left(x_{n}\right)}\right)=C_{2} e_{n}^{2}+2 e_{n}^{3}\left(C_{3}-C_{2}^{2}\right)-e_{n}^{4}\left(3 C_{2}^{3}-7 C_{2} C_{3}+4 C_{4}\right)+(O) e_{n}^{5}$

Derivando dos veces la ecuación (19) y sustituyendo en la ecuación de (8):

$f^{\prime}\left(y_{n}\right)=2 C_{2} e_{n}+6 e_{n}^{2}\left(C_{3}-C_{2}^{2}\right)-4 e_{n}^{3}\left(3 C_{2}^{3}-7 C_{2} C_{3}+4 C_{4}\right)+(O) e_{n}^{4}$

$f^{\prime \prime}\left(y_{n}\right)=2 C_{2}+12 e_{n}\left(C_{3}-C_{2}^{2}\right)-12 e_{n}^{2}\left(3 C_{2}^{3}-7 C_{2} C_{3}+4 C_{4}\right)+(0) e_{n}^{3}$

Desarrollado en series de Taylor el numerador de (8):

$\beta_{1} f\left(y_{n}\right)\left(f^{\prime}\left(y_{n}\right)\right)^{2}=8 C_{2}^{3} e_{n}^{4}+64 C_{2}^{2} e_{n}^{5}\left(C_{3}-C_{2}\right)+(0) e_{n}^{6}$

El denominador de (8) desarrollado en series de Taylor es igual a:

$$
\begin{aligned}
\beta_{2}\left(f\left(y_{n}\right)\right)^{2}+\beta_{3} \alpha_{x}\left(f\left(y_{n}\right)\right)^{3} f^{\prime \prime}\left(y_{n}\right) & \\
= & 8 C_{2}^{2} e_{n}^{2}+48 C_{2} e_{n}^{3}\left(C_{3}-C_{2}^{2}\right)+8 e_{n}^{4}\left(29 C_{2}^{4}-46 C_{2}^{2} C_{3}+8 C_{2} C_{3}+9 C_{3}^{2}\right)+(O) e_{n}^{5}
\end{aligned}
$$

Dividiendo (22) entre (23):

$$
\begin{gathered}
\frac{\beta_{1} f\left(y_{n}\right)\left(f^{\prime}\left(y_{n}\right)\right)^{2}}{\beta_{2}\left(f\left(y_{n}\right)\right)^{2}+\beta_{3} \alpha_{x}\left(f\left(y_{n}\right)\right)^{3} f^{\prime \prime}\left(y_{n}\right)}=\frac{8 C_{2}^{3} e_{n}^{4}+64 C_{2}^{2} e_{n}^{5}\left(C_{3}-C_{2}\right)+(O) e_{n}^{6}}{8 C_{2}^{2} e_{n}^{2}+48 C_{2} e_{n}^{3}\left(C_{3}-C_{2}^{2}\right)+8 e_{n}^{4}\left(29 C_{2}^{4}-46 C_{2}^{2} C_{3}+8 C_{2} C_{3}+9 C_{3}^{2}\right)+(O) e_{n}^{5}} \\
=C_{2} \mathrm{e}_{n}^{2}+2 \mathrm{e}_{\mathrm{n}}^{3}\left(C_{3}-C_{2}^{2}\right)-\frac{\mathrm{e}_{n}^{4}\left(41 \mathrm{C}_{2}^{4}-70 \mathrm{C}_{2}^{2} \mathrm{C}_{3}+8 \mathrm{C}_{2} \mathrm{C}_{4}+21 \mathrm{C}_{3}^{2}\right)}{\mathrm{C}_{2}}-\frac{4 \mathrm{e}_{n}^{5}\left(\mathrm{C}_{3}-\mathrm{C}_{2}^{2}\right)\left(32 \mathrm{C}_{2}^{4}-61 \mathrm{C}_{2}^{2} \mathrm{C}_{3}+2 \mathrm{C}_{2} \mathrm{C}_{4}+27 \mathrm{C}_{3}^{2}\right)}{\mathrm{C}_{2}^{2}}
\end{gathered}
$$

De donde se tiene finalmente que el método representado por las ecuaciones (7) y (8) es de cuarto orden:

$$
\begin{aligned}
& x_{n+1}= y_{n}-\frac{f\left(y_{n}\right)\left(f^{\prime}\left(y_{n}\right)\right)^{2}}{\left(f\left(y_{n}\right)\right)^{2}+\left(f\left(y_{n}\right)\right)^{3} f^{\prime \prime}\left(y_{n}\right)}=C_{2} e_{n}^{2}+\left(2 C_{3}-2 C_{2}^{2}\right) e_{n}^{3}+(O) e_{n}^{4}- \\
&\left(C_{2} e_{n}^{2}+2 e_{n}^{3}\left(C_{3}-C_{2}^{2}\right)-\frac{e_{n}^{4}\left(41 C_{2}^{4}-70 C_{2}^{2} C_{3}+8 C_{2} C_{4}+21 C_{3}^{2}\right)}{C_{2}}-\frac{4 e_{n}^{5}\left(C_{3}-C_{2}^{2}\right)\left(32 C_{2}^{4}-61 C_{2}^{2} C_{3}+2 C_{2} C_{4}+27 C_{3}^{2}\right)}{C_{2}^{2}}\right) \\
& \boldsymbol{e}_{n+1}=\frac{e_{n}^{4}\left(41 C_{2}^{4}-70 C_{2}^{2} C_{3}+8 C_{2} C_{4}+21 C_{3}^{2}\right)}{C_{2}}+\frac{4 e_{n}^{5}\left(C_{3}-C_{2}^{2}\right)\left(32 C_{2}^{4}-61 C_{2}^{2} C_{3}+2 C_{2} C_{4}+27 C_{3}^{2}\right)}{C_{2}^{2}}+(O) e_{n}^{6}
\end{aligned}
$$

La aplicación del método propuesto en este trabajo consiste en resolver $(N-1)$ funciones no lineales, en las que se debe satisfacer la condición: $f i\left(V_{1}, V_{2}, V_{N}\right) \approx 0$, para $i=1, N$ (Villafuerte et al., 2013). Para cada nodo $i$, exceptuando el nodo compensador, se genera una función $f_{i}\left(V_{1}, V_{2}, V_{N}\right)$ de la forma expresada por la ecuación (6). En dónde: $N$, es el número de nodos de la red eléctrica, $\overline{Y_{l}}$ la admitancia entre el nodo $i$ y el nodo $j, \bar{V}_{l}$ el voltaje del nodo $i, P_{i}$ la potencia real neta y $Q_{i}$ la potencia reactiva neta en el nodo $i$, respectivamente. En los nodos de voltaje controlado, la potencia reactiva Qi (Saadat, 1999) se calcula en cada iteración con la ecuación (26). 
$Q_{i}^{(k)}=-I m\left(V_{i}^{(k-1) *}\left[\sum_{j=1}^{i-1} Y_{i j} V_{j}^{(k)}+\sum_{j=i}^{N} Y_{i j} V_{j}^{(k-1)}\right]\right)$

En cada nodo y en cada iteración $k$ se calcula la potencia reactiva con la ecuación (26), se ajusta la magnitud del voltaje y se actualiza su ángulo. En la ecuación (26) $k$, es el contador de iteraciones, y * significa el conjugado. En la figura 1, (Saadat, 1999) se muestra el diagrama unifilar de un sistema de potencia de cinco nodos, para su análisis se establecen las funciones $f\left(x_{n}\right)$ con los voltajes de cada nodo y se aplica el método representado por las ecuaciones (7) y (8). Con los datos mostrados en la red de la figura 1 , el nodo uno, es el nodo compensador en él se conoce su magnitud y su ángulo. Los nodos dos y tres, son nodos de voltaje controlado en donde se conoce la magnitud del voltaje, pero se desconoce su ángulo y se desconoce también el valor de la potencia reactiva $Q_{i}$, la que se calcula con la ecuación (26), se sustituye en las ecuaciones (6) para evaluar las funciones $\mathrm{fi}\left(\mathrm{V}_{2}, \mathrm{~V}_{3}, \mathrm{~V}_{4}, \mathrm{~V}_{5}\right)$, y determinar iterativamente los voltajes con el método de dos pasos aquí propuesto.

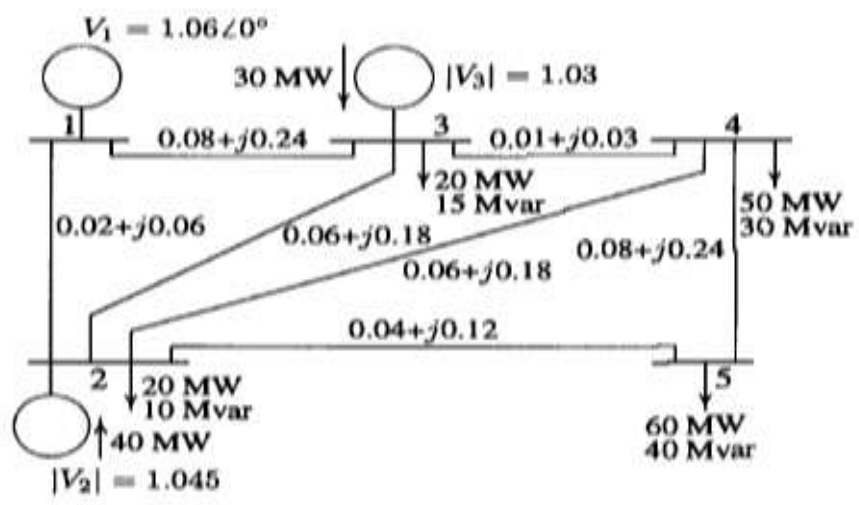

Fig. 1: Red de cinco nodos (Saadat, 1999)

El método aquí propuesto, consiste en generar las funciones $f i\left(V_{1},,, V_{5}\right)$, calcular la primera derivada fi' $\left(V_{1},,,, V_{5}\right)$ y su segunda derivada fi' $\left(V_{1},,, V_{5}\right)$ con respecto al voltaje de cada nodo y determinar iterativamente su valor. Los nodos dos y tres de la figura 1 son de voltaje controlado, se mantiene constante su magnitud y se calcula iterativamente su ángulo, en los nodos de carga se calcula iterativamente la magnitud y el ángulo el voltaje. Con los datos de la figura 1, (Saadat, 1999), las admitancias Yij están contenidos en la matriz de admitancias $Y$ de la ecuación (27).

$Y=\left[\begin{array}{lllll}Y_{11} & Y_{12} & Y_{13} & Y_{14} & Y_{15} \\ Y_{21} & Y_{22} & Y_{23} & Y_{24} & Y_{25} \\ Y_{31} & Y_{32} & Y_{33} & Y_{34} & Y_{35} \\ Y_{41} & Y_{42} & Y_{43} & Y_{44} & Y_{45} \\ Y_{51} & Y_{52} & Y_{53} & Y_{54} & Y_{55}\end{array}\right]$

Con las admitancias $Y_{i j}$ de la ecuación (27) se generan las funciones representadas por las ecuaciones (28), (29), (30) y (31).

$$
\begin{aligned}
& f_{2}\left(V_{1}, V_{2}, \ldots V_{N}\right)=P_{2}^{(k)}-j Q_{2}^{(k)}-V_{2}^{*}\left(Y_{21} V_{1}^{(k)}+Y_{22} V_{2}^{(k)}+Y_{23} V_{3}^{(k)}+Y_{24} V_{4}^{(k)}+Y_{25} V_{5}^{(k)}\right) \\
& f_{3}\left(V_{1}, V_{2}, \ldots V_{N}\right)=P_{3}^{(k)}-j Q_{3}^{(k)}-V_{3}^{*}\left(Y_{31} V_{1}^{(k)}+Y_{32} V_{2}^{(k)}+Y_{33} V_{3}^{(k)}+Y_{34} V_{4}^{(k)}+Y_{35} V_{5}^{(k)}\right) \\
& f_{4}\left(V_{1}, V_{2}, \ldots V_{N}\right)=P_{4}^{(k)}-j Q_{4}^{(k)}-V_{4}^{*}\left(Y_{41} V_{1}^{(k)}+Y_{42} V_{2}^{(k)}+Y_{43} V_{3}^{(k)}+Y_{44} V_{4}^{(k)}+Y_{45} V_{5}^{(k)}\right) \\
& f_{5}\left(V_{1}, V_{2}, \ldots V_{N}\right)=P_{5}^{(k)}-j Q_{5}^{(k)}-V_{5}^{*}\left(Y_{51} V_{1}^{(k)}+Y_{52} V_{2}^{(k)}+Y_{53} V_{3}^{(k)}+Y_{54} V_{4}^{(k)}+Y_{55} V_{5}^{(k)}\right)
\end{aligned}
$$

Con la formulación utilizada en este trabajo cualquier nodo puede ser el compensador (Villafuerte R. et al., 2013). Las funciones representadas por las ecuaciones (28) a (31), con su primera y segunda derivada 
respecto al voltaje Vi y la matriz de admitancias (27), son utilizadas en la aplicación del método aquí propuesto. Los datos iniciales que se consideran para las variables (voltajes) del sistema de la figura 1 son los siguientes (Saadat, 1999):

$V_{1}=1.06\left[0 ; V_{2}^{(o)}=1.045\left[0 ; V_{3}^{(o)}=1.03\left[0 ; V_{4}^{(o)}=1.0\left[0 ; V_{5}^{(o)}=1.0\lfloor 0\right.\right.\right.\right.$ valor por unidad

La ecuación (32) es utilizado para terminar el proceso iterativo es.

$\varepsilon=\left|\sqrt{\sum_{1}^{N}\left(\frac{f_{i}\left(V_{1}, V_{2}^{(k)},, V_{N}^{(k)}\right)}{f_{i}^{\prime}\left(V_{1}, V_{2}^{(k)},,, V_{N}^{(k)}\right)}\right)^{2}}\right| \leq t o l$

La solución iterativa de las funciones (28) a (31) del sistema de la figura 1, (Saadat, 1999), con su primera y segunda derivada calcula los voltajes de cada nodo, con los que se obtiene el flujo de potencia. En los nodos de voltaje controlado, en cada iteración se ajusta la magnitud del voltaje y se actualiza su ángulo (Grainger et al., 1996). Si se rebasan los límites de potencia reactiva en la iteración k, se asigna el límite violado y se considera como nodo de carga en esa iteración.

\section{RESULTADOS}

Se analizan cinco sistemas de potencia que se encuentran en la literatura, para los dos primeros se muestran los valores de voltajes obtenidos, en los sistemas de 14,30 y 57 nodos, se representa gráficamente el comportamiento del número de iteraciones con la constante $\beta_{0}$ de la ecuación (7). La simulación digital se lleva a cabo formando la función no lineal en cada nodo de la red y se aplica el método representado por las ecuaciones (7) y (8) hasta que el criterio de convergencia (32) se cumpla, lo que implica que la función: $\mathrm{f}_{\mathrm{i}}\left(\mathrm{V}_{2}, \mathrm{~V}_{3}, \mathrm{~V}_{4}, \mathrm{~V}_{5}, \ldots \mathrm{V}_{\mathrm{N}}\right) \approx 0$, para $\mathrm{i}=2,$, , $\mathrm{N}$.

Como primer caso, con los datos de la figura 1 (Saadat, 1999), se lleva a cabo la simulación digital considerando una tolerancia de $1.0 \mathrm{e}-04$, se obtienen los voltajes en diez iteraciones y se muestran en la tabla 1. En la segunda columna se muestra la magnitud del voltaje de cada nodo, en la tercera columna se proporciona el ángulo de ese voltaje, y en la cuarta columna se muestra el valor de la función de voltaje de cada nodo. En la quinta y sexta columna se proporciona la magnitud del voltaje y su ángulo, que son reportados por Saadat (1999).

Tabla 1: Voltajes de cada nodo del sistema de la figura 7.9 (Saadat, 1999)

\begin{tabular}{cccccccc}
\hline \multicolumn{3}{c|}{ Ecuaciones (7) y (8) $(10$ iteraciones) } & \multicolumn{2}{c}{ (Saadat, 1999) } \\
\hline Nodo & $|\mathrm{V}| \mathrm{pu}$ & $\left\llcorner\mathrm{V}^{\circ}\right.$ & $f(\mathrm{Vi})$ & $|\mathrm{V}| \mathrm{pu}$ & $\left\llcorner\mathrm{V}^{\circ}\right.$ \\
\hline 1 & 1.06 & 0 & 0 & 1.06 & 0 \\
2 & 1.0450 & -1.7825 & 0.000293 & 1.045 & -1.782 \\
3 & 1.0300 & -2.6640 & 0.001586 & 1.030 & -2.664 \\
4 & 1.0186 & -3.2431 & 0.000570 & 1.019 & -3.243 \\
5 & 0.9901 & -4.4051 & 0.000399 & 0.990 & -4.405 \\
\hline
\end{tabular}

El segundo caso se encuentra en Anderson et al., (2003), que corresponde a un sistema de potencia de nueve nodos. Los valores de las variables (magnitudes de voltajes y ángulos) obtenidos con el método propuesto son mostrados en la tabla 2 (columnas dos y tres), los valores reportados en la misma fuente se muestran en la quinta columna y en la sexta columna. En la cuarta columna se proporciona el valor de la función $\mathrm{f}_{\mathrm{i}}\left(\mathrm{V}_{2}, \mathrm{~V}_{3}, \mathrm{~V}_{4}, \mathrm{~V}_{5}, \mathrm{~V}_{6}, \mathrm{~V}_{7}, \mathrm{~V}_{8}, \mathrm{~V}_{9}\right)$, que puede ser utilizada como error para terminar el proceso iterativo.

Los sistemas de prueba del IEEE de 14, 30 y 57 nodos se simulan con el método propuesto para analizar su comportamiento con respecto a la constante $\beta_{0}$ de la ecuación (7), el número de iteraciones en cada caso es mostrado en las figuras 2, 3 y 4 . La figura 2 muestra el comportamiento del número de iteraciones con la constante $\beta_{0}$ de la ecuación (7), para los tres sistemas de prueba se considera una tolerancia de 0.01 .

El tiempo de ejecución para los tres casos representados en la figura 2, fue en general de 15.625 milisegundos. La figura 3 muestra el comportamiento del número de iteraciones con la constante $\beta_{\circ}$ de la ecuación (7), para los tres sistemas de prueba se considera una tolerancia de 0.001 . 
Tabla 2: Valores de cada variable (voltaje) del sistema de la figura 2.

\begin{tabular}{cccccccc}
\hline \multicolumn{5}{c|}{ Ecuaciones 7 y 8 (14 iteraciones) } & \multicolumn{2}{c}{$|\mathrm{V}|$ (Anderson et al., 2003) } \\
\hline Nodo & $|\mathrm{V}| \mathrm{pu}$ & $\left\llcorner\mathrm{V}^{\circ}\right.$ & $f(\mathrm{Vi})$ & $|\mathrm{V}| \mathrm{pu}$ & $\left\llcorner\mathrm{V}^{\circ}\right.$ \\
\hline 1 & 1.04 & 0 & 0 & 1.04 & 0 \\
2 & 1.0250 & 9.2801 & 0.000916 & 1.0250 & 9.2797 \\
3 & 1.0250 & 4.6659 & 0.000511 & 1.0250 & 4.6645 \\
4 & 1.0258 & -2.2167 & 0.001317 & 1.0258 & -2.2168 \\
5 & 0.9956 & -3.9886 & 0.000663 & 0.9956 & -3.9889 \\
6 & 1.0127 & -3.6873 & 0.000961 & 1.0127 & -3.6875 \\
7 & 1.0258 & 3.7201 & 0.000534 & 1.0258 & 3.7194 \\
8 & 1.0159 & 0.7277 & 0.000030 & 1.0159 & 0.7273 \\
9 & 1.0324 & 1.9668 & 0.000684 & 1.0324 & 1.9665 \\
\hline
\end{tabular}

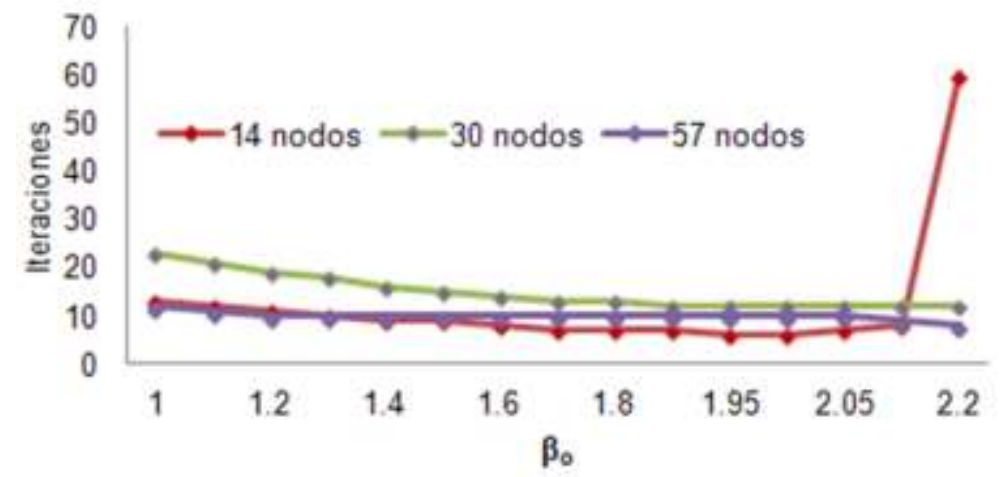

Fig. 2: Comportamiento de los tres sistemas de prueba con respecto a $\beta_{0}$ con tolerancia de 0.01

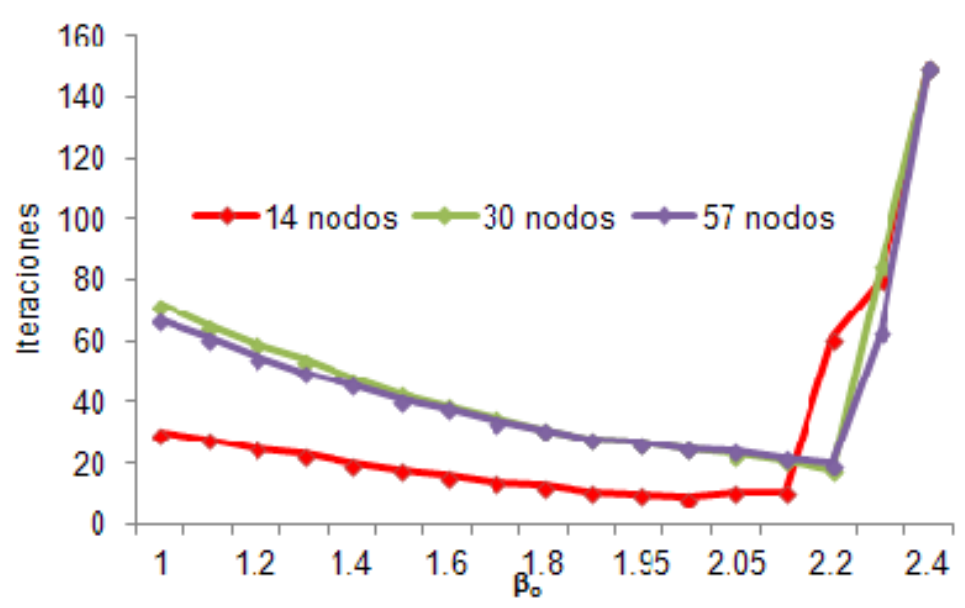

Fig. 3: Comportamiento de los tres sistemas de prueba con respecto a $\beta_{0}$ con tolerancia de 0.001

El tiempo de ejecución para los tres casos representados en la figura 3, fue 15.625 milisegundos para los sistemas de 14 y 30 nodos y para el sistema de 57 nodos oscilo ente 15.625 y 31.25 milisegundos. La figura 4 muestra el comportamiento del número de iteraciones con la constante $\beta_{\circ}$ de la ecuación (7), para los tres sistemas de prueba se considera una tolerancia de 0.0001 .

Los tiempos de ejecución para los tres casos mostrados en la figura 4, oscilaron entre 15.625 y 31.25 milisegundos. Si la constante $\beta_{0}$ es mayor a 2.1, el tiempo de ejecución puede ser mayor a los 90 milisegundos o se presenta una divergencia del método. La constante $\beta_{o}$, es en realidad un factor de aceleración que se puede elegir entre 1.8 y 2.2, para que el método propuesto converja en la menor cantidad de iteraciones, como es mostrado en las figuras 2, 3 y 4. 


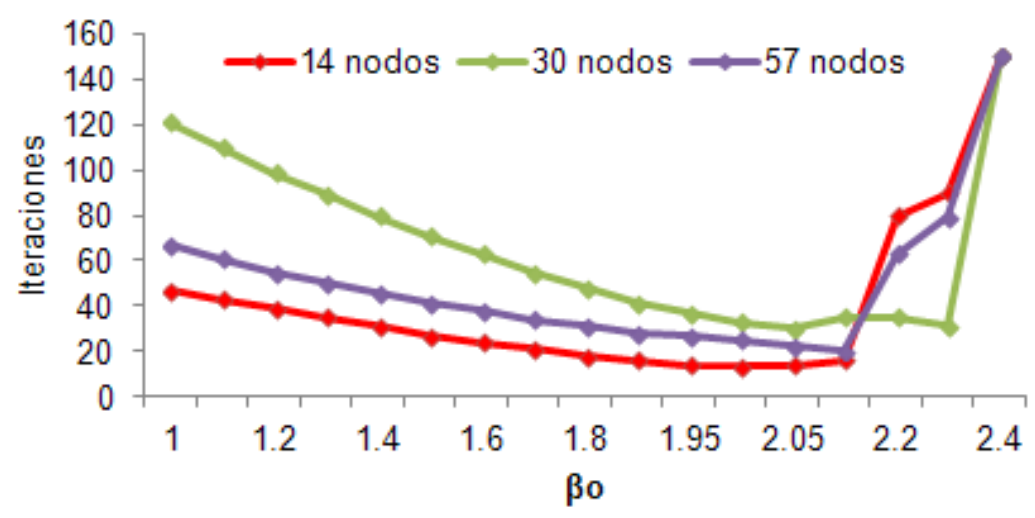

Fig. 4: Comportamiento de los tres sistemas de prueba con respecto a $\beta_{\text {o }}$ con tolerancia de 0.0001

\section{CONCLUSIONES}

El método propuesto se ideó con el objetivo de tener una opción más para calcular flujos potencia. EI método consiste principalmente en la formación de la función $\mathrm{f}_{\mathrm{i}}\left(\mathrm{V}_{1},,, \mathrm{~V}_{\mathrm{N}}\right)$ y sus derivadas para cada nodo. Este procedimiento se lleva a cabo en cada iteración y requiere únicamente del manejo de operaciones elementales. Debido a ello, no implica de una programación complicada, aunque si es necesario el uso de esquemas de almacenamiento de la matriz de admitancias para sistemas de 30 nodos o más. El método propuesto no intenta competir con ninguna versión del método de Newton-Raphson, como se mencionó anteriormente, representa una opción que tiene como desventaja requerir de un mayor número de iteraciones. Sin embargo, tiene las ventajas de no utilizar métodos de especiales de factorización ni de una lógica sofisticada de programación.

\section{REFERENCIAS}

Anderson P. M. y A. A. Fouad, Power System Control and Stability, 37-38, IEEE Press Power Engineering Series, New York, NY (1994)

Bahgat Mohamed S.M. y Hafiz M.A., Three-Step Iterative Method with Eighteenth Order Convergence for Solving Nonlinear Equations, doi: 10.12732/ijpam.v93i1.7, International Journal of Pure and Applied Mathematics, Volume 93 No. 1, 85-94 (2014)

Chapra, S.C. y Canale R.P., Numerical Methods for Engineers, McGraw-Hill Higher Education (2014)

Chun C. y B. Neta, A Third-Order Modification of Newton's Method for Multiple Roots, Applied Mathematics and Computation, $211474-479$ (2009)

Grainger J.J. y W.D. Stevenson Jr., Análisis de Sistemas de Potencia, 315-330, McGraw-Hill/Interamericana de México (1996)

Hale H. V. y R. W. Goodrich, Digital computation of power flow-some new aspects, Trans. AIEE (Power Apparatus and Systems), vol. 78A, p. 919, (1959).

Homeier H.H.H, On Newton-Type Methods with Cubic Convergence, Journal of Computational and Applied Mathematics 205 1-5, (2005)

Hueso J., E. Martínez, C. Teruel, Determination of Multiple Roots of Nonlinear Equations and Applications, doi: 10.1007/s10910-014-0460-8, J Math Chem, 53:880-892 (2015)

Kou Jisheng, Li Yitian, Wang Xiuhua, Third-Order Modification of Newton's Method, Journal of computational and applied mathematics 205, 1-5 (2007)

Mamdouh Abdel-Akher, and Ali Selim, Mohamed M. Aly, Initialised load-flow analysis based on Lagrange polynomial approximation for efficient quasi-static time-series simulation, doi: 10.1049/iet-gtd.2015.0866 2768-2774 (2015)

Mohamed S. M. Bahgat, New Two-Step Iterative Methods for Solving Nonlinear Equations, Journal of Mathematics Research, Vol., 4, No 3 (2012)

Nakamura S., Métodos Aplicados con Software, Prentice Hall Hispanoamericana de México (1992) 
Nayak Nivedita, Dr. A.K. Wadhwani, Performance of Newton-Raphson Techniques in Load Flow Analysis using MATLAB, National Conference on Synergetic Trends in engineering and Technology (STET-2014) International Journal of Engineering and Technical Research ISSN: 2321-0869, Special Issue (2014)

Petko D. Proinov y Stoil I. Ivanov, On the Convergence of Halley's Method for Multiple Polynomial Zeros, doi: 10.1007/s00009-014-0400-7, Springer Basel (2014)

Ramandeep Behl V. Kanwar, Highly efficient classes of Chebyshev-Halley type methods free from secondorder derivative, Proceedings of 2014 RAECS UIET Panjab University Chandigarh, 06-08 (2014)

Saadat H. Power Systems Analysis, 2a edition, 224-232, 295-296, McGraw-Hill Series in Electrical and Computer Engineering, New York, NY (1999)

Sauer, P.M., Explicit load flow series and functions, IEEE Trans., PAS-100, pp. 3754-3763 (1981)

Stagg G. y A.H. El-Abiad, Computer Methods in Power System Analysis, 270-276, McGraw Hill, (1968)

Stott, B., y ALSAC, 0., Fast decoupled load flow, IEEE, Trans., PAS-93, pp. 859-869 (1974)

Tinney W.F., y Clifford E.H., Power Flow Solution by Newton's method, IEEE Transactions on Power Apparatus and Systems, vol. pas-86, no. 11 (1967)

Tewarson R., Sparse Matrices, 72-75, Academic Press, New York, NY (1973)

Villafuerte R., R.A. Villafuerte S., Jesús Medina C., Edgar Mejía S., Multi-Step Methods Applied to Nonlinear Equations of Power Networks, doi: 10.5923/j.eee.20130305.02, Electrical and Electronic Engineering, 3(5), 128-132 (2013)

W. Xu, Y. Liu, J. C. Salomon, T.LE y G. W. K. Chang, Series load flow: A novel non-iterative load flow method, IEE Proc Generation Transmission \& Distribution, vol. 145, No.3, May (1988)

Zhu J., Optimization of Power System Operation, John Wiley and Sons, Hoboquen, New Jersey (2009) 